\title{
Cognitive Side Effects of Antiepileptic Drugs
}

\author{
Kimford J. Meador
}

\begin{abstract}
All of the established antiepileptic drugs (AEDs) can produce cognitive side effects, which are increased with polypharmacy and with increasing dosage and anticonvulsant blood levels. However, cognitive side effects are usually modest for AED monotherapy with anticonvulsant blood levels within the standard therapeutic ranges. Further, these effects are offset in part by reduced seizure activity. Controversy exists regarding possible differential cognitive effects of AEDs. A large portion of the literature examining the comparative cognitive effects of AEDs is limited by inadequate study designs. When these design flaws are considered, there is no convincing evidence of clinically significant differences in cognitive side effects of AEDs except possibly for bromide, phenobarbital and benzodiazepines. The role of cognitive side effects should be kept in proper perspective when choosing AED therapy. The cognitive side effects of anticonvulsant drugs may be overt but many times are rather subtle. It is important though to be able to recognize these effects and to put them into perspective as to how they affect our patients.
\end{abstract}

Résumé: Effets secondaires cognitifs des anticonvulsivants. Tous les médicaments antiépileptiques (MAEs) peuvent produire des effets secondaires cognitifs qui sont amplifiés par la polypharmacie et l'augmentation de la posologie et des niveaux sanguins. Cependant, les effets secondaires cognitifs des MAEs utilisés en monothérapie sont habituellement légers lorsque les taux sanguins demeurent dans la zone thérapeutique standard. De plus, ces effets sont contrebalancés partiellement par une diminution des crises. La possibilité qu'il existe des différences entre les effets secondaires cognitifs des MAEs est controversée. La validité d'une grande partie de la littérature qui compare les effets cognitifs des MAEs est limitée par le fait que les schémas d'études sont inadéquats. Quand on tient compte de cette restriction, il n'existe pas d'évidence convainquante qu'il y a des différences cliniques significatives dans les effets secondaires cognitifs des MAEs, exception faite peut-être des bromures, du phénobarbital et des benzodiazépines. Le rôle des effets secondaires cognitifs devrait être gardé en perspective quand on choisit un MAE.

Can. J. Neurol. Sci. 1994; 21: Suppl. 3-S12-S16

\section{Historical Review}

The first person to write in detail about cognitive issues in epilepsy was probably Lennox. ${ }^{1}$ He pointed out that different variables contribute to cognitive dysfunction in patients with epilepsy, including genetic factors, the epilepsy itself, brain abnormalities acquired prior to the epilepsy, pathological sequelae from the seizures, and psychosocial issues. Lennox also raised the issue that the antiepileptic drugs (AEDs) themselves could cause cognitive problems. He wrote that "many physicians, in attempting to extinguish seizures, only succeed in drowning the finer intellectual processes of their patients."'

In a ironic twist, AED cognitive side effects may be viewed as actually leading to the discovery of the first true anticonvulsant. Prior to its use as an anticonvulsant, bromide had been noted by Huette to produce general sedation, mental slowing, and depression of sexuality. ${ }^{2}$ Subsequently, multiple publications confirmed the untoward cognitive effects of bromide, although these effects were never formally investigated.
However, it was the depression of sexuality that led to the discovery of the anticonvulsant properties of bromide. Since hysterical epilepsy was believed at that time to be secondary to masturbation, Locock suggested that bromide might be efficacious. ${ }^{3}$ Of course when bromide was used for that purpose, it was subsequently discovered that bromide was quite a good anticonvulsant for true epilepsy. ${ }^{4}$

The very first study actually designed as a systematic investigation of the cognitive side effects of AEDs was by SomerfeldZiskind and Ziskind in $1940 .^{5}$ They randomized 100 patients into two groups. One group was treated with phenobarbital, and the control group was treated with a ketogenic diet. The patients were followed for 1 - 2 years with repeated neuropsychological

From the Section of Behavioral Neurology, Department of Neurology, Medical College of Georgia, Augusta.

Reprint requests to: Kimford J. Meador, Section of Behavioral Neurology, Department of Neurology, Medical College of Georgia, Augusta, Georgia, USA 30912-3208 
testing. A marked reduction in seizures occurred in the phenobarbital group, but no differences in cognitive side effects were found between the two groups.

That same year, Lennox published the results of his large survey of several thousand patients with epilepsy. ${ }^{1}$ In a subset of that study, he reported on the effects of phenobarbital, bromides, and "patent drugs", which were mixtures of various drugs, mostly made up of phenobarbital and bromide. His data showed that both phenobarbital and bromide were effective AEDs, which produced a marked reduction in seizures. More interesting were the patients' subjective reports on mentality. Only a minority of patients experienced a subjective deterioration in cognition on AED therapy. Cognition remained unchanged in $58 \%$ on phenobarbital and in $57 \%$ on bromides. In fact, $30 \%$ on phenobarbital and $26 \%$ on bromides reported improved cognition. This study was a survey and did not involve formal neuropsychological testing, but from the patients' perception, most felt no adverse effects of AEDs on cognition.

Where these early studies reliable, and did they reflect a real phenomenon? I believe that the answer is yes. Phenobarbital and bromide are probably two of the most sedating of the AEDs, yet when they are used in monotherapy at doses that give anticonvulsant blood levels within standard therapeutic ranges, their effects on cognition are rather modest. Further, these effects are offset in part by the improvement in cognition produced by reduced seizure activity.

\section{Modern Studies}

Subsequently, there has been a plethora of studies examining the cognitive effects of anticonvulsants, and two facts are very well established. ${ }^{6}$ The first one is that decreasing the number of AEDs improves cognition and frequently decreases seizures..$^{7-12}$ In fact, the increase in adverse effects from AED polypharmacy may be seen across a variety of side effects. Second, as you increase the dose and thus the anticonvulsant blood levels, there is an increasing risk of developing cognitive side effects. ${ }^{13-23}$ This is especially true as the anticonvulsant blood levels rise above the recommended standard therapeutic ranges.

In contrast, the issue of differential cognitive effects for AEDs remains somewhat controversial. Several drugs have been suggested to be superior in this regard. ${ }^{24}$ Although there are some differences, these differences appear to be rather subtle and of dubious clinical significance. ${ }^{6} \mathrm{~A}$ variety of different experimental techniques has been used to examine the cognitive effects of AEDs. Designs have included monotherapy or multidrug studies with parallel groups and/or repeated measures as the AEDs are added or withdrawn. Across the major AEDs, a mixture of results has been obtained. ${ }^{6.25}$ Another experimental design employs healthy volunteers. The advantage of this design is that it removes the confounding effects of cerebral damage and of the seizures themselves. Again, a mixture of results has been obtained across carbamazepine, phenytoin, and valproate. ${ }^{6,25}$ More consistent adverse effects have been noted with phenobarbital and benzodiazepines in the healthy volunteer studies. ${ }^{6.25}$

One of the best designs to examine the cognitive effects of AEDs is the crossover monotherapy study, in which each patient is treated and tested on each drug. Thus, the variance produced by individual variability is controlled. One of the most famous of these studies is the one by Dodrill and Troupin, on which they actually published two articles several years apart. ${ }^{26,27}$

The first article, in 1977, stated that carbamazepine produced fewer side effects than phenytoin. ${ }^{28}$ However, the mean anticonvulsant blood level for the carbamazepine condition was $9.3 \mu \mathrm{g} / \mathrm{mL}$ (therapeutic range $=6-12$ ), but the mean anticonvulsant blood level for the phenytoin condition was $31.2 \mu \mathrm{g} / \mathrm{mL}$ (therapeutic range $=10-20$ ). So, in reality, this article actually compared performance on one drug in mid-therapeutic range with another drug at grossly toxic levels. When Dodrill and Troupin reanalyzed their data in 1991, controlling for anticonvulsant blood levels, they found no cognitive differences between the two drugs. ${ }^{27}$

The reason for such a diversity in results across studies is because many of the studies have ignored critical factors in their design and analysis. ${ }^{6}$ In addition, the differences are small if present at all. Design flaws have included the following. Some studies have not randomly selected patients. For example, patients who are taking one of two drugs are selected from the clinic, and then they are tested and compared. However, they were not randomly assigned to the drugs in the first place, so that group differences may exist as a function of selection bias. Another poor design is the use of non-orthogonal contrasts. A control group is tested once and compared with a group of patients who are repeatedly tested as they are tapered off AEDs. Thus, test-retest effects are confounded with the drug effects. Some studies fail to control for seizures, and others have not controlled for the blood levels, concomitant medications or the duration of treatment. Further, the characteristics of the cognitive tests vary across studies. Several studies have ignored Type I error and conducted multiple analyses without controlling for chance statistical findings. Finally, the magnitude of some statistically significant differences may be such that the differences are not clinically significant.

\section{Studies by the MCG Group}

In an attempt to address some of these issues and to design a study that avoided such pitfalls, our group at the Medical College of Georgia (MCG) conducted two investigations. ${ }^{28,29}$ The first study was in patients with epilepsy and employed a double-blind crossover design. ${ }^{28}$ Each subject was treated with each drug for 3 months, and the order of therapy was randomized. The drugs included carbamazepine, phenobarbital, and phenytoin. Anticonvulsant blood levels and seizure frequency were obtained to control for these factors. Cognitive testing was performed at the end of each 3 month drug treatment period. The neuropsychological battery was chosen to include tests that have proven sensitive to AED effects in prior studies, and to include standard neuropsychological tests that have proven clinical utility for the assessment of cognitive deficits from a variety of etiologies. The battery assessed attention, verbal learning, simple motor speed, fine coordinated motor speed, graphomotor coding speed, decisional/motor speed, cognitive processing speed, and affective symptoms. The battery yielded 15 variables for each condition.

Analyses of covariance controlling for both seizures and anticonvulsant blood levels revealed only one statistically significant difference between drugs. Performance on the digit 
symbol modality test (a graphomotor coding task) was impaired in subjects on phenobarbital compared with the two other AEDs. Mean performances for phenytoin and carbamazepine were exactly the same. A follow-up analysis was conducted across all of the cognitive variables to rule out significant individual variation by examining the number of instances in which individuals had a difference of greater than 1 standard deviation between their performance on different drugs. On 14 occasions, carbamazepine was worse than phenytoin. On 10 occasions, phenytoin was. worse than carbamazepine. These results fail to suggest a difference between these two drugs. On 28 occasions, phenobarbital was worse than carbamazepine and phenytoin, suggesting that overall there was greater impairment with phenobarbital compared with the other two drugs.

In the second study, the comparative effects of carbamazepine and phenytoin were re-examined. ${ }^{29}$ This study employed healthy adults. Use of these subjects allowed a more intensive and sensitive cognitive battery than could be given to patients with epilepsy. It also eliminated the confounding cognitive effects of seizures. As in the first study, a randomized double-blind crossover design was used. Subjects were tested at baseline and after 1 month of therapy on the first drug. They were then tapered off the first drug, washed out over a month, placed on the second drug, treated for a month, and tested on the second drug at the end of the month. Finally, the subjects were tapered off the second drug, washed out over a month, and tested again at the very end of the study for a second baseline. Thus, there were two baseline assessments off drugs in addition to the assessments on each of the two drugs. The neuropsychological battery included tests of verbal learning, visual-spatial learning, vigilance, reading speed/interference, simple motor speed, fine coordinated motor speed, decisional/motor speed, cognitive processing speed, graphomotor coding speed and scales for affective and subjective symptoms.

The resulting 20 variables per condition were subjected to analyses comparing the two AEDs, controlling for blood levels, and comparing the drugs with the non-drug condition. There were only two statistical differences for the drug-drug comparisons. One was for simple motor speed (i.e., finger-tapping) in favor of carbamazepine, and the other was for reading speed/interference (i.e., Stroop) in favor of phenytoin. These differences were rather small and could have been due possibly to Type 1 error, considering the number of comparisons performed. Comparison of the non-drug baseline conditions with the drug conditions revealed statistical differences for eight variables (i.e., Stroop, grooved pegboard, choice reaction time, Hopkins subjective checklist, and four measures from the Profile of Mood States). Again, these differences were not very large in magnitude. The results from the P3 event-related potential offer some insight into the size of drug effects. The P3 is an endogenously invoked potential that provides an index of cognitive processing speed. The P3 latency is slowed by dementia, trauma, aging, and a variety of pharmacologic agents. An overthe-counter antihistamine like chlorpheniramine can produce an approximately 25 millisecond slowing in the P3 latency. ${ }^{30}$ In contrast, the P3 slowing produced by AED monotherapy in mid-therapeutic ranges was about 12 milliseconds in the above study.

One surprising finding in our second study was the lack of AED effects on two standard tests of anterograde memory (i.e., Complex Figures and Selective Reminding Test) ${ }^{29}$ However,
AED effects were seen on another test of memory. In a subset of the patients, spectral components of EEG were measured during resting and activated states. ${ }^{31}$ Small but statistically significant differences in the spectral EEG components were produced by the AEDs. More robust drug effects were seen for performance on the activation task, which consisted of a verbal paragraph with subsequent free recall. In the non-drug conditions, subjects recalled $60-65 \%$ of the information in the paragraphs. The AEDs reduced recall to about $50 \%$, but there was no difference between the two AEDs. The greater sensitivity of this memory task to AED effects may be due to a greater attentional component, since the individual bits of information to be recalled are presented only once.

\section{Perspective on MCG Studies}

The results from our studies need to be put into perspective with other well-controlled studies, especially in regard to the relative weaknesses of these two studies. First, although the repeated measures design adds a great deal of statistical strength, the sample sizes have been criticized as being small. In this regard, the large Veterans Administration (VA) Cooperative Study, which had over 400 patients, should be examined..$^{32}$ The study investigated the cognitive effects of four major AEDs (carbamazepine, phenobarbital, phenytoin, and primidone) in patients with new-onset epilepsy. Compared with matched controls, the patients with epilepsy had poorer neuropsychological performance before they were put on AEDs. This difference was much larger than any effect of the AEDs. In fact, the overall cognitive performances pre- and post-initiation of AEDs were quite small as depicted in the graph in the article. One might ask if individual AED differences might be obscured by the grand averages. However, we examined the actual means of the neuropsychological tests for each AED group and found no suggestion of any statistically significant, much less any clinically significant, differences across the drugs in the cognitive battery used in the VA study. ${ }^{25}$ Recently, a second VA Cooperative Study comparing carbamazepine and valproate was completed and found no differences in cognitive side effects. ${ }^{33}$

Another design problem raised in regard to our studies was the length of therapy. These were short studies lasting only a few weeks or months. Could AED effects possibly build up over time to produce a delayed insidious encephalopathy? Dodrill and Wilensky investigated this issue. ${ }^{34}$ They performed repeated neuropsychological evaluations over 5 years in patients on phenytoin monotherapy, phenytoin with one or more drugs, or AED regimens exclusive of phenytoin. These patients were well controlled; their AED dosages and blood levels remained stable. There was no deterioration in cognition of these patients over the 5 years.

\section{Cognitive Effects in Different Age Groups}

Another important issue is whether AEDs may exhibit differnt cognitive effects across age. The issue is less resolved because there have been fewer AED cognitive studies in children. Further, the effect of AEDs on learning and memory may be more critical in this age group. However, a review of some recent studies suggests that the differential effects of AEDs in children may be similar to adults.

The only double-blind crossover study in children was conducted by Vining et al. ${ }^{35}$ They compared the effects of phenobarbital and valproate in children with epilepsy and found that performance was poorer on phenobarbital for several measures. 
Farwell et al. examined the effects of phenobarbital in children with febrile seizures using a parallel design. ${ }^{36}$ Patients were treated with phenobarbital or placebo for 2 years. At the end of that time, the patient group treated with phenobarbital had lower IQs compared with the placebo group. The AEDs were then withdrawn and patients retested 6 months later. The phenobarbital group improved 5 points, and the placebo group 2 points. This difference was still statistically significant, so Farwell et al. raised the question as to whether long-term effects of phenobarbital persisted. It should be noted that there was a 4 point improvement in a matched group of healthy controls, so that the 5 point improvement in the phenobarbital could not be attributed solely to drug withdrawal. Although the findings of this study are probably valid, some controversies have been raised. One is that the analysis was based on intention to treat, and only $76 \%$ of patients were tested at the end of the 2 years. Further, only $44 \%$ were still on the double-blind at the time of testing.

Forsythe et al. investigated children with new onset partial or generalized tonic-clonic seizures. ${ }^{37}$ Patients were randomized to carbamazepine, phenytoin, or valproate. All of the children completing the 1 year study were seizure free, and their anticonvulsant blood levels were in the low to low therapeutic range. A battery of neuropsychological tests was conducted before AED therapy, and then again 1 year after the beginning of AED monotherapy. There were not many differences on most of the tests, but some differential effects were seen on a series of five memory tests. The carbamazepine group was significantly worse than the valproate group. The phenytoin group fell in between the other two drugs, not being statistically different than either of the other two drugs.

At the other end of the age spectrum, the elderly have increased susceptibility to the cognitive effects of a variety of drugs. Only one recent study has specifically examined the differential cognitive effects of AEDs in the elderly. Craig and Tallis compared the effects of phenytoin and valproate in elderly patients with new-onset epilepsy. ${ }^{38}$ The cognitive changes compared with the non-drug baselines and the differences in cognitive performances between the two AEDs were minimal.

\section{Conclusion}

All the established AEDs can produce cognitive side effects. Several new AEDs are in various stages of development and approval. Some of these drugs appear to have few cognitive side effects, but it remains to be seen using appropriately designed studies with direct comparison with other AEDs whether these effects are statistically or clinically significant. Thus, it is unclear whether an AED can be designed that reduces neuronal irritability without reducing neuronal excitability. However, this ideal should remain a goal in the development of new AEDs.

The risks of cognitive side effects definitely increase with polytherapy and with increasing dose/anticonvulsant blood levels. On monotherapy within standard therapeutic ranges, the cognitive side effects are modest, and they are offset in part by reduced seizures. There is no convincing evidence for clinically siopificant differences in coonitive side_effects of AFPs expept
AED therapy is based on a balance of risks and benefits. For the individual patient, the best AED (and the best dose) is the one that provides the best seizure control with the fewest side effects.

\section{REFERENCES}

1. Lennox WG. Brian injury, drugs and environment as causes of mental decay in epilepsy. Am J Psychiatry 1942; 99: 174-180.

2. Huette C. Recherches sur les propriétés physiologiques et thérapeutiques de bromure de potassium. Memoirs de la Société de Biologie 1850; 2: 19.

3. Locock C. Discussion of paper by EH Seiveking. Analysis of 42 cases of epilepsy observed by the author. Lancet 1857; 1 : 527 528.

4. Voisin A. De l'Emploi de Bromure de Potassium Dans les Maladies Nerveuses. Paris, 1875.

5. Somerfeld-Zisking E, Ziskind E. Effect of phenobarbital on the mentality of epileptic patients. Arch Neurol Psychiatry 1940; 43: 70-79.

6. Meador KJ, Loring DW. Cognitive effects of antiepileptic drugs. In: Devinsky O, Theodore WH, eds. Epilepsy and Behavior. New York: Wiley-Liss, 1991: 151-170.

7. Milano Collaborative Group of Studies on Epilepsy. Long-term intensive monitoring in the difficult patient: preliminary results of 16 months of observations - usefulness and limitations. In: Gardner-Thorpe C, ed. Antiepileptic Drug Monitoring. Tunbridge Wells, Kent: Pitman Medical, 1977: 197-213.

8. Schain RJ, Ward JW, Guthrie D. Carbamazepine as an anticonvulsant in children. Neurology 1977; 27: 476-480.

9. Shorvon SD, Reynolds EH. Reduction in polypharmacy for epilepsy. Br Med J 1979; 2: 1023-1025.

10. Thompson PJ, Trimble MR. Anticonvulsant drugs and cognitive functions. Epilepsia 1982; 23: 531-544.

11. Ludgate J, Keating J, O'Dwyer R, Callaghan N. An improvement in cognitive function following polypharmacy reduction in a group of epileptic patients. Acta Neurol Scand 1985; 71: 448452.

12. Prevey ML, Mattson RH, Cramer JA. Improvement in cognitive functioning and mood state after conversion to valproate monotherapy. Neurology 1989; 39: 1640-1641.

13. Tchicaloff $M$, Gaillard F. Quelques effects indesirables des medicaments anti-epileptiques, sur le rendements intellectuels. Revue de Neuropsychiatrie Infantile et d'Hygiene Mentale de l'Enfance 1970; 18: 599-602.

14. Reynolds EH, Travers RD. Serum anticonvulsant concentrations in epileptic patients with mental symptoms. A preliminary report. Br J Psychiatry 1974; 124: 440-445.

15. Dekaban AS, Lehman EJ. Effects of different dosages of anticonvulsant drugs on mental performances in patients with chronic epilepsy. Acta Neurol Scand 1975; 52: 319-330.

16. Dodrill CB. Diphenylhydantoin serum levels, toxicity, and neuropsychological performance in patients with epilepsy. Epilepsia 1975; 16: 593-600.

17. MacLeod CM, Dekaban AS, Hunt E. Memory impairment in epileptic patients: selective effects of phenobarbital concentration. Science 1978; 202: 1102-1104.

18. Camfield CS, Chaplin S, Doyle AB, et al. Side effects of phenobarbital in toddlers: behavioral and cognitive aspects. J Pediatr 1979; 95 : 361-365.

19. Thompson P, Huppert FA, Trimble $M$. Phenytoin and cognitive function: effects on normal volunteers and implications for epilepsy. Br J Clin Psychol 1981; 20 (Pt 3): 155-162.

20. Thompson PJ, Trimble MR. Sodium valproate and cognitive functioning in normal volunteers. Br J Clin Pharmacol 1981; 12: 819-824

21. Thompson PJ, Trimble MR. Anticonvulsant serum levels: relationship to impairments of cognitive functioning. J Neurosurg Psychiatry 1983: 46: 227-233 
23. Gillham RA, Williams N, Wiedmann $\mathrm{K}$, et al. Concentration-effect relationships with carbamazepine and its epoxide on psychomotor and cognitive function in epileptic patients. J Neurol Neurosurg Psychiatry 1988; 51: 929-933.

24. Trimble MR. Neurobehavioral effects of anticonvulsants [editorial]. JAMA 1991; 265: 1307-1308.

25. Nichols ME, Meador KJ, Loring DW. Neuropsychological effects of antiepileptic drugs: a current perspective. Clin Neuropharmacol: In press.

26. Dodrill CB, Troupin AS. Psychotropic effects of carbamazepine in epilepsy: a double-blind comparison with phenytoin. Neurology 1977; 27: 1023-1028.

27. Dodrill CB, Troupin AS. Neuropsychological effects of carbamazepine and phenytoin: a reanalysis. Neurology 1991; 41: 141143.

28. Meador KJ, Loring DW, Huh K, Gallagher BB, King DW. Comparative cognitive effects of anticonvulsants. Neurology 1990; 40: 391-394.

29. Meador KJ, Loring DW, Allen ME, et al. Comparative cognitive effects of carbamazepine and phenytoin in healthy adults. Neurology 1991; 41: 1537-1540.

30. Meador KJ, Loring DW, Thompson EE, Thompson WO. Differential cognitive effects of terfenadine and chlorpheniramine. J Allergy Clin Immunol 1989; 84: 322-325.

31. Meador KJ, Loring DW, Abney OL, et al. Effects of carbamazepine and phenytoin on EEG and memory in healthy adults. Epilepsia 1993; 34: 153-157.
32. Smith DB, Mattson RH, Cramer JA, et al. Results of a nationwide Veterans Administration Cooperative Study comparing the efficacy and toxicity of carbamazepine, phenobarbital, phenytoin, and primidone. Epilepsia 1987; 28 (Suppl 3): S50-S58.

33. Prevey ML, Delaney RC, Cramer JA, et al. Valproate versus carbamazepine: comparison of effects on cognitive functioning [abstract]. Epilepsia 1992; 33 (Suppl 3): 110.

34. Dodrill CB, Wilensky AJ. Neuropsychological abilities before and after 5 years of stable antiepileptic drug therapy. Epilepsia 1992; 33: 327-334.

35. Vining EPG, Mellitis ED, Dorsen MM, et al. Psychologic and behavioral effects of antiepileptic drugs in children: a doubleblind comparison between phenobarbital and valproic acid. Pediatrics 1987; 80: 165-174.

36. Farwell JR, Lee YJ, Hirtz DG, et al. Phenobarbital for febrile seizures - effects on intelligence and on seizure recurrence. $\mathrm{N}$ Engl J Med 1990; 322: 364-369.

37. Forsythe I, Butler R, Berg I, McGuire R. Cognitive impairment in new cases of epilepsy randomly assigned to carbamazepine, phenytoin, and sodium valproate. Dev Med Child Neurol 1991; 33: 524-534.

38. Craig I, Tallis R. The impact of sodium valproate and phenytoin on cognitive function in elderly patients: results of a single-blind randomized comparative study. Epilepsia 1994; 35: 381-390. 